\title{
IrRECIST Partial Response
}

National Cancer Institute

\section{Source}

National Cancer Institute. irRECIST Partial Response. NCI Thesaurus. Code C140315.

A decrease of $30 \%$ or more in TMTB relative to baseline, non-target lesions are irNN, and no unequivocal progression of new non-measurable lesions. 